\section{Kann Montelukast die Prednisolon-Therapie bei akuten Asthmaanfällen vermeiden?}

In den letzten Jahren hat Montelukast eine wichtige Rolle in der Dauertherapie des milden chronischen Asthmas errungen. Unklar ist, welche Rolle es in der Behandlung akuter Asthmaanfälle spielen kann.

\begin{abstract}
anadische Wissenschaftler behandelten in einer randomisierten doppelblinden Studie 130 Kinder zwischen 2 und 17 Jahren mit milden bis moderaten akuten Asthmaanfällen. In den Notfallambulanzen wurden die Kinder mit Prednisolon über maximal 8 Stunden stabilisiert. Nach der Entlassung bekamen sie dann eine Behandlung über fünf Tage entweder mit Prednisolon oder Montelukast. Als primärer Erfolgsparameter wurde ein Scheitern der Therapie innerhalb der ersten acht Tage angesehen.

Bei den Ergebnissen zeigte sich mit $7,9 \%$ eine niedrigere Rate von Therapie-
\end{abstract}

versagern in der Prednisolon-Gruppe gegenüber $22,4 \%$ in der Montelukast-Gruppe. Dies betraf vor allem jüngere Patienten. In der Montelukast-Gruppe war häufiger eine zusätzliche Therapie mit Prednisolon erforderlich. Die Autoren schließen aus ihren Untersuchungen, dass in der Therapie des akuten Asthmas Montelukast Prednisolon nicht ersetzen kann.

Kommentar: Das Ergebnis widerspricht einer älteren Studie an Patienten, die nicht in der Klinik behandelt werden mussten. Bei diesen war ein Effekt von Montelukast auch beim akuten Asthma gesehen worden. Insgesamt zeigen die Ergebnisse der aktuellen Studie, dass es keine gute Alternative zur entzündungshemmenden und schleimhautabschwellenden Wirkung systemischer Steroide bei akuten Asthmaanfällen gibt. Dies trifft besonders auf die engen Atemwege von Kleinkindern zu. Da eine kurzfristige Therapie mit Kortikosteroiden in der Regel auch keine langfristigen Risiken für die Kinder mit sich bringt, sollte diese bei den Patienten, die deutlich beeinträchtigt sind, weiterhin allererste Wahl sein. In der Dauertherapie spielt Montelukast den Vorteil der geringeren Langzeitnebenwirkungen aus.

Dr. Martin Claßen

Schuh S et al. Can montelukast shorten prednisolone therapy in children with mild to moderate Acute asthma? A randomized controlled trial. J Pediatr 2009; 155: 795800

\title{
Die Rolle der vorsätzlich Ungeimpften
}

Im Jahr 2008 kam es in San Diego, USA, zu einem Ausbruch von Masern. Ein sieben Jahre alter ungeimpfter Junge war maserninkubiert von einer Reise in die Schweiz zurückgekehrt. Der Fall wurde von den Centers for Disease Control and Prevention analysiert.

$\mathrm{N}$ achdem der Junge aus der Schweiz zurückgekehrt war, kam es zum größten Masernausbruch in San Diego seit 1991. Zunächst erkrankte der Junge selbst. Dies zog insgesamt elf weitere Fälle von Masern nach sich - durchweg bei ungeimpften Kindern. Vom Indexfall selbst wurden acht Kinder infiziert, die dann wiederum drei weitere Kinder ansteckten. 839 Personen wurden exponiert, von denen 106 keine sichere Masernimmunität hatten. Bei 86 war mit einem nennenswerten Risiko der Übertragung zu rechnen. Die Eltern dieser Kinder hatten häufig die Impfung zum üblichen Zeitpunkt abgelehnt.

Die Infektionskette konnte dann dank konsequenter Maßnahmen unterbrochen werden: Die Kinder, welche nicht geimpft werden konnten, entweder, weil die Eltern dies weiterhin ablehnten oder weil sie zu jung waren, wurden unter eine 21-tägige Quarantäne gestellt. Die übrigen Kinder wurden geimpft. Für die Bekämpfung der
Infektionsausbreitung fielen pro Einzelfall im öffentlichen Sektor, d. h. ohne Berücksichtigung z. B. von Verdienstausfällen, Kosten in Höhe von 10.376 US-\$. Besonders aufschlussreich ist der Hinweis auf die Ursachen der Verweigerung einer Impfung. Es ergaben sich Cluster von Wohnbezirken, in denen Impfgegner besonders häufig anzutreffen waren. Hier waren Bildungsgrad und Durchschnittseinkommen der Eltern höher als der Durchschnitt. Der Hauptgrund für die Ablehnung der Impfung waren Sorge vor Nebenwirkungen und die Überzeugung, die Krankheit sei nicht so gefährlich.

Kommentar: Man erkennt Ähnlichkeit zu den Verhältnissen in Deutschland. Auch bei uns sind es häufig die sogenannten besseren Leute mit akademischem Hintergrund, die ihre Kinder gar nicht oder nicht zeitgerecht gegen Masern impfen lassen wollen. Eine gesetzliche Pflicht zur Impfung ist gewiss nicht sinnvoll.

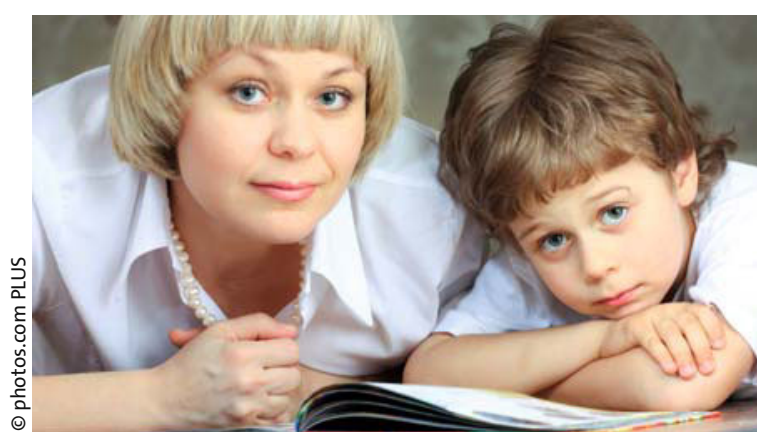

Vor allem Eltern mit einem höheren Bildungsgrad und höherem Einkommen ließen ihre Kinder seltener gegen Masern impfen.

Man muss aber drüber nachdenken, Impfverweigerer zu verpflichten, die Kosten zu übernehmen, welche bei einem Ausbruch entstehen. Auf unsere Verhältnisse übertragen ist daher auch mit wenigstens $7.000 €$ pro Folgefall von $\mathrm{Ma}-$ sern zu rechnen. Alternativ zu einer gesetzlichen Pflicht könnte daher die Pflicht zum Abschluss einer Haftpflichtversicherung für diese Kosten in Betracht kommen.

Dr. Hartmut Koch

Sugerman DE et al. Measles outbreak in a highly vaccinated population, San Diego, 2008: role of the intentionally unvaccinated. Pediatrics 2010; 125: 747-55 